Publ. RIMS, Kyoto Univ.

12 Suppl. (1977), 351-355.

\title{
Existence and Continuation of Holomorphic Solutions of Partial Differential Equations
}

\author{
by
}

Ph. Pallu de la Barriere*

\begin{abstract}
Necessary conditions and sufficient conditions are given for the existence and the continuation of holomorphic solutions of partial differential equations near the characteristic boundary of an open subset of $\boldsymbol{C}^{n}$.**
\end{abstract}

\section{$\S 1$. Definitions and Results}

Let $\Omega$ be an open subset of $M=C^{n+1}$ defined by the equation:

$$
\Omega=\left\{z \in C^{n+1} \mid \varphi(z)>0\right\}
$$

where $\varphi$ is a real analytic function with real values and $d \varphi(z) \neq 0$ when $\varphi(z)=0$.

If $\mathcal{O}$ designates the sheaf of holomorphic functions on $M$, we denote by $\mathcal{O}^{+}$the sheaf on $N=\partial \Omega$ defined by this stalk at $z \in N$ :

$$
\mathcal{O}_{z}^{+}=\underset{z \in \omega}{\lim } \mathcal{O}\left(\omega^{+}\right), \omega^{+}=\omega \cap \Omega
$$

where $\omega$ runs over a fundamental system of neighbourhoods of $z$ in $M$.

Let $P$ be a differential operator with holomorphic coefficients defined in a neighbourhood of $N$ :

$$
P\left(z, D_{z}\right)=\sum_{|\alpha| \leqq m} a_{\alpha} D_{z}^{\alpha} .
$$

We give necessary conditions and sufficient conditions to have one of the following properties:

$(P R)_{z}$ Continuation: $f \in \mathcal{O}_{z}^{+}$and $P f \in \mathcal{O}_{z} \Rightarrow f \in \mathcal{O}_{z}$

$(E X)_{z}$ Existence: $\forall g \in \mathcal{O}_{z}^{+},{ }^{\exists} f \in \mathcal{O}_{z}^{+}$so that $P f=g$

Received September 7, 1976.

* Collège de France, Paris. 24 av. de l'Observatoire, 75014, Paris, France.

** An integral version of this paper has appeared in [3]. 
If $N$ is non-characteristic with respect to $P$ at $z$, it is known that we have the properties $(P R)_{z}$ and $(E X)_{z}([5],[1])$.

If $N$ is simply characteristic with respect to $P$ at $z, Y$. Tsuno ([1]) has given a geometric sufficient condition and a necessary condition to have the property $(P R)_{z}$.

We will compare later his conditions with ours.

Later, we shall need the $\bar{\partial}_{b}$-system of tangential Cauchy-Riemann equations on $N$. We recall its definition briefly.

The problem being local, we can suppose that $\frac{\partial \varphi}{\partial \bar{z}_{0}} \neq 0$ near $z=\left(z_{0}, \cdots\right.$, $\left.z_{n}\right)$. We can define $\bar{\partial}_{b}$ on $N$ by:

$$
\bar{\partial}_{b} u=0 \Leftrightarrow X_{j} u=0, \quad j=1, \cdots, n,
$$

where $X_{j}=\frac{\partial}{\partial \bar{z}_{j}}-\frac{\partial \varphi}{\partial \bar{z}_{j}}\left(\frac{\partial \varphi}{\partial \bar{z}_{0}}\right)^{-1} \frac{\partial}{\partial \bar{z}_{0}}$.

If we denote by $\Sigma$ the real characteristic variety of the system $\bar{\partial}_{b}$ in $S^{*} N, \Sigma$ is a reunion of two cotangent vector fields $\Sigma^{+}$and $\Sigma^{-}$where

$$
\Sigma^{+}(z)(z, \xi(z)) \in S^{*} N, \xi_{j}(z)=-i \frac{\partial \varphi}{\partial \bar{z}_{j}}(z), \quad j=0, \cdots, n,
$$

$\Sigma^{-}$is the antipodal of $\Sigma^{+}$in $S^{*} N$.

Now we define an operator on $N$ associated with $P$. Let $\left(Y_{j}\right)$, $j=0, \cdots, n$, be a family of $n+1$ complex vector fields on $N$ defined by

$$
Y_{j}=\frac{\partial}{\partial z_{j}}-\frac{\partial \varphi}{\partial z_{j}}\left(\frac{\partial \varphi}{\partial \bar{z}_{0}}\right)^{-1} \frac{\partial}{\partial \bar{z}_{0}}
$$

We have $\left[Y_{j}, Y_{k}\right]=0, \forall j, k=0, \cdots, n$ and the family $Y_{j}, j=0, \cdots, n$ is linearly independent, so we can define the operator $P_{b}$ on $N$ by:

$$
P_{b}=\sum_{|\alpha| \leq m} \widetilde{a}_{\alpha} Y^{\alpha} \quad \text { where } \quad \widetilde{a}_{\alpha}=\left.a_{\alpha}\right|_{N} .
$$

Note that:

$N$ is characteristic with respect to $P$ at $z \Leftrightarrow \sigma\left(P_{b}\right)\left(\Sigma^{+}(z)\right)=0$ (where $\sigma(Q)$ designates the principal symbol of a differential operator $Q$ ).

Let $p_{b}=0$ be a reduced equation of the complex characteristic variety of the operator $P_{b}$.

Definition. The generalized Levi-form of $(\Omega, P)$ at $z$, denoted by 
$L_{z}$, is the hermitian form on $\boldsymbol{C}^{n+1}$ defined by

$$
\begin{aligned}
\tau \in C^{n+1}, & L_{z}(\tau)=\sum_{1 \leq j, k \leq n}\left\{\sigma\left(X_{k}\right), \overline{\sigma\left(X_{j}\right)}\right\}\left(\Sigma^{+}(z)\right) \tau_{k} \bar{\tau}_{j} \\
& +2 \operatorname{Re} \sum_{1 \leq j \leq n}\left\{\sigma\left(X_{j}\right), \bar{p}_{b}\right\}\left(\Sigma^{+}(z)\right) \tau_{j} \tau_{n+1} \\
& +\left\{p_{b}, \bar{p}_{b}\right\}\left(\Sigma^{+}(z)\right)\left|\tau_{n+1}\right|^{2}
\end{aligned}
$$

where $\{f, g\}$ designates the Poisson bracket of two homogeneous functions on $S^{*} M$.

Suppose that $N$ is characteristic for $P$ at $z$, that is:

$$
\sigma(P)(z, d \varphi(z))=0
$$

then we have the following:

Theorem I. If there is $\tau \in C^{n+1}$ so that $L_{z}(\tau)<0$, we have the property $(P R)_{z}$.

Theorem II. If $\Omega$ is strictly pseudo-convex at $z$ we have $\operatorname{det} L_{z}>0 \Rightarrow(E X)_{z}$ and no $(P R)_{z}$, $\operatorname{det} L_{z}<0 \Rightarrow(P R)_{z}$ and no $(E X)_{z}$.

Remark I. After a change of coordinate near $z^{0} \in N$ which transforms the function $\varphi$ in $\Psi$ defined by

$$
\Psi(z)=\operatorname{Im} z_{0}+\sum_{1 \leq j, k \leq n} \frac{\partial^{2} \varphi}{\partial z_{j} \partial \bar{z}_{k}}\left(z^{0}\right) z_{j} \bar{z}_{k}
$$

the generalized Levi-form has the same signature as that of the hermitian form

$$
\begin{aligned}
-L_{z^{0}}(\tau) & =\sum_{1 \leq j, k \leq n} \frac{\partial^{2} \varphi}{\partial z_{j} \partial \bar{z}_{k}}\left(z^{0}\right) \tau_{j} \bar{\tau}_{k} \\
& -2 \operatorname{Im}_{1 \leq j \leq n} \sum_{(j)}\left(z^{0}, d \varphi\left(z^{0}\right)\right) \tau_{j} \bar{\tau}_{n+1} \\
& +\sum_{1 \leq j, k \leq n} p^{(j)}\left(z^{0}, d \varphi\left(z^{0}\right)\right) \cdot \overline{p^{(k)}\left(z^{0}, d \varphi\left(z^{0}\right)\right)} \frac{\partial^{2} \varphi}{\partial z_{j} \partial \bar{z}_{k}}\left(z^{0}\right)\left|\tau_{n+1}\right|^{2} .
\end{aligned}
$$

Here we have used the usual notations 


$$
p_{(j)}=\frac{\partial p}{\partial z_{j}}, \quad p^{(j)}=\frac{\partial p}{\partial \xi_{j}}
$$

Remark II. (Y. Tsuno's result) Suppose that the normal of $N$ at $\approx$ is simply characteristic and denote by $(z(\tau), \xi(\tau))$ the complex bicharacteristic curve of $P$ issued from $(z, d \varphi(z))$. The result of [4] which is comparable with ours is:

If there is $\tau_{0} \in \mathbb{C}$ so that $\left.\frac{d^{2}}{d t^{2}} \varphi\left(z\left(t \tau_{0}\right)\right)\right|_{t=0}<0(t \in \mathbb{R})$ then we have the property $(P R)_{z}$.

In fact that is exactly:

If there is $\tau=\left(\tau_{0}, \cdots, \tau_{n+1}\right) \in C^{n+1}$ so that:

$$
\forall j=1, \cdots, n, \tau_{j}=\tau_{n+1} \cdot p^{(j)}\left(z^{0}, d \varphi\left(z^{0}\right)\right) \text { and } L_{z}(\tau)<0
$$

we have $(P R)_{z}$.

Remark III. By geometrical arguments, we can show that the condition to have the property $(P R)_{z}$ can be applied if only $\varphi$ is in the class of $C^{3}$.

\section{$\S$ 2. Sketch of the Proof}

We prove these results in two steps. To begin with we show that $(P R)_{z}$ and $(E X)_{z}$ are equivalent to properties of the sheaf of microfunction solutions of an induced system of differential equations on $N$. It is a consequence of a more general result of Kashiwara-Kawai [2], but we give a direct elementary proof and we calculate explicitly the induced system. More precisely the first result is the following:

If $\tilde{\mathscr{L}}$ designates the sheaf on $N$ of the differential operators and $\tilde{\mathscr{C}}$ the sheaf on $S^{*} N$ of microfunctions, if $\mathscr{M}_{\left(\bar{\partial}_{b}, P_{b}\right)}$ designates the $\widetilde{\mathscr{D}}$-module associated to the system of differential equations $\left(\bar{\partial}_{b}, P_{b}\right)$, we have

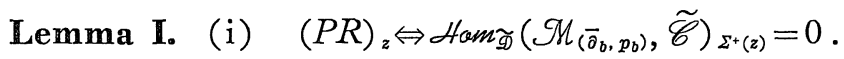

(ii) If $\Omega$ is strictly pseudo-convex at $z$, we have $(E X)_{z} \Leftrightarrow \mathcal{E} x t \widetilde{\mathscr{D}}^{1}\left(\mathcal{M}_{\left(\bar{\partial}_{b}, p_{b}\right)}, \widetilde{\mathscr{C}}\right)_{\Sigma+(z)}=0$.

The second step is to study the vanishing of the group $\mathcal{E}_{x} \widetilde{\mathscr{D}}^{k}\left(\mathcal{M}_{\left(\bar{\partial}_{b}, P_{b}\right)}\right.$, 
$\widetilde{\mathscr{C}})_{\Sigma^{+}(z)}$. For this we make use of the structure theorem of [S.K.K] for a system of microdifferential equations which has a non involutive real characteristic variety.

We show that the generalized Levi-form of the system $\left(\bar{\partial}_{b}, P_{b}\right)$ in the sense of [S.K.K] is $L_{z}$. The only difficulty is to prove the following:

Lemma II. The compex characteristic variety $S S\left(\mathcal{M}_{\left(\bar{\partial}_{b}, P_{b}\right)}\right)$ of the system $\left(\bar{\partial}_{b}, P_{b}\right)$ is defined by the equation

(1) $S S\left(\mathscr{M}_{\left(\bar{a}_{b}, P_{b}\right)}\right)=\left\{z^{*} \in P^{*} Y \mid \sigma\left(X_{1}\right)\left(z^{*}\right)=\cdots=\sigma\left(X_{n}\right)\left(z^{*}\right)=p_{b}\left(z^{*}\right)=0\right\}$ where $P^{*} Y$ is the projective bundle of a complexification $Y$ of $N$.

In fact we have in general:

$$
S S\left(\mathcal{M}_{\left(\bar{\partial}_{b}, P_{b}\right)}\right) \subset V
$$

where $V$ denotes the right hand side of (1).

To prove $V \subset S S\left(\mathcal{M}_{\left(\bar{o}_{b}, P_{b}\right)}\right)$, we must prove that:

if $z^{*} \in V, \forall Q_{0}, \cdots, Q_{n} \in \widetilde{\varepsilon}_{z^{*}}$, we have

$$
\sum Q_{i} \widehat{X}_{i}+Q_{0} \widehat{P}_{b}=1_{\widetilde{\mathcal{E}}}
$$

where $\widehat{R}$ denotes the complexification of $R \in \widetilde{D}$ and $\widetilde{\mathcal{E}}$ the sheaf on $P^{*} Y$ of the microdifferential operators ([S.K.K $]$ ).

To prove this, using the Frobenius theorem, we find a local coordinate of $Y, Z_{1}, \cdots, Z_{2 n+1}$ so that $\widehat{X}_{i}$ is transformed to $\frac{\partial}{\partial Z_{i}} \forall i=1, \cdots, n$, and in this situation we prove the lemma 2 using the symbolic calculus on micro-differential operators of [S.K.K].

\section{References}

[1] Bony, J. M. and Schapira, P., Existence et prolongement des solutions holomorphes des equations aux derivées partielles, Inv. Math. 17 (1972), 95-105.

[2] Kashiwara, M. and Kawai, T., On the boundary values of elliptic system of partial differential equations 1, Proc. Japan Acad., 48 (1970), 712-715.

[3] Pallu de la Barriere, P., Existence et prolongement des solutions holomorphes des equations aux derivées partielles, J. Math. Pures et Appl. 55 (1976), 21-46.

[S.K.K] Sato, M., Kawai, T. and Kashiwara, M., Microfunctions and pseudo-differential equations, Lecture notes in Math., 287 (1971), 265-524.

[4] Tsuno, Y., On the continuation of holomorphic solutions of characteristic differential equations, J. Math. Soc. Japan, 26, 3 (1974), 523-548.

[5] Zerner, M., Domaine d'holomorphie des fonctions verifiant une equation aux derivées partielles, C. R. Acad. Sc. Paris, Serie A, 272 (1971), 1646-1648. 
